\title{
NAFLD fibrosis score può essere usato nei servizi ambulatoriali per identificare complicanze vascolari croniche oltre alla fibrosi epatica avanzata nel diabete tipo 2
}

\author{
Sara Sterpetti ${ }^{1} \cdot$ Raffaella Buzzetti $^{1}$
}

Accettato: 21 dicembre 2020 / Pubblicato online: 12 agosto 2021

(c) The Author(s) 2021

Commento a:

NAFLD fibrosis score (NFS) can be used in outpatient services to identify chronic vascular complications besides advanced liver fibrosis in type 2 diabetes.

R. Lombardi, L. Airaghi, G. Targher, G. Serviddio, G. Maffi, A. Mantovani, C. Maffeis, A. Colecchia, R. Villani, L. Rinaldi, E. Orsi, G. Pisano, L.E. Adinolfi, S. Fargion, A.L. Fracanzani.

J Diabetes Complications (2020) 34(11):107684

La steatosi epatica non alcolica (NAFLD), presente in circa il 60-75\% dei pazienti con diabete tipo 2 (DM2), può essere rilevata tramite la misurazione non invasiva della rigidità epatica con strumento dedicato, il Fibroscan [1].

Data la difficoltà di utilizzare routinariamente il Fibroscan, è stato sviluppato il NAFLD fibrosis score (NFS), che predice il rischio di fibrosi epatica utilizzando dati anamnestici (età, Body Mass Index, presenza di diabete) e dati clinici (valori di transaminasi, piastrine e albuminemia).

In un precedente studio [2] Lombardi e collaboratori avevano dimostrato che la presenza di fibrosi epatica, valutata con Fibroscan, si associava a un aumentato rischio di complicanze vascolari croniche in pazienti con DM2.

In questo studio osservazionale multicentrico gli autori hanno valutato l'associazione tra fibrosi epatica e complicanze croniche nel DM2, utilizzando sia NFS che il Fibroscan. Sono stati arruolati 394 pazienti con DM2, di cui il $19 \%$ presentava una complicanza macro-vascolare $(14 \%$ pregresso IMA, $4 \%$ stroke, $1 \%$ entrambi) mentre il $33 \%$ presentava una o più complicanze micro-vascolari $(26 \%$ nefropatia, $14 \%$ retinopatia, $6 \%$ neuropatia periferica).

$凶$ R. Buzzetti

raffaella.buzzetti@uniroma1.it

1 Dipartimento di Medicina Sperimentale, Sapienza Università di Roma, Roma, Italia
Tutti i pazienti venivano sottoposti a ecografia epatica e, in caso di riscontro di NAFLD, veniva eseguito screening per fibrosi epatica, utilizzando sia NFS sia il Fibroscan. La fibrosi epatica avanzata veniva diagnosticata se NFS $\geq 0,676$ e in caso di rigidità epatica al Fibroscan $\geq 8,7 \mathrm{kPa}$ e $\geq 7,2$ $\mathrm{kPa}$ per le sonde M/XL. L'89\% dei pazienti presentava un quadro di NAFLD e, tra questi, è stata individuata fibrosi epatica nel $22 \%$, utilizzando NFS, nel $14 \%$ utilizzando il Fibroscan.

Stratificando la popolazione in base al cutoff di NFS $\geq 0,676$, i pazienti con fibrosi risultavano più anziani e presentavano un Body Mass Index maggiore. Nella popolazione con fibrosi è stata rilevata una maggiore prevalenza di complicanze cardiovascolari e di neuropatia periferica (31 vs $15 \%$ e 19 vs $3 \%$, rispettivamente $p<0,001)$. Un maggior uso di metformina e DPP4-inibitori (DPP4i) è stato osservato nella coorte priva di fibrosi.

NFS $\geq 0,676$ rimaneva significativamente associato con la neuropatia periferica (OR corretto 4,9; CI 95\% 1,4-17,1, $p=0,01)$ e parzialmente con lo stroke ischemico (OR corretto 3,5; CI 95\% 0,95-14,2, $p=0,07$ ) dopo correzione per centro, sesso, tabagismo, durata di malattia, compenso glicemico, ipertensione e attuale terapia con metformina, DPP4i, ACE-inibitori/sartani o statine.

Risultati sovrapponibili sono stati ottenuti stratificando la popolazione sulla base dei cut-off del Fibroscan.

In conclusione, NFS si è dimostrato uno strumento facilmente utilizzabile nella pratica clinica e sovrapponibile al Fibroscan per individuare pazienti con DM2 a rischio di sviluppare fibrosi epatica e complicanze croniche micro/macro-vascolari.

Funding Note Open access funding provided by Università degli Studi di Roma La Sapienza within the CRUI-CARE Agreement. 
Nota della casa editrice Springer Nature rimane neutrale in riguardo alle rivendicazioni giurisdizionali nelle mappe pubblicate e nelle affiliazioni istituzionali.

Open Access This article is licensed under a Creative Commons Attribution 4.0 International License, which permits use, sharing, adaptation, distribution and reproduction in any medium or format, as long as you give appropriate credit to the original author(s) and the source, provide a link to the Creative Commons licence, and indicate if changes were made. The images or other third party material in this article are included in the article's Creative Commons licence, unless indicated otherwise in a credit line to the material. If material is not included in the article's Creative Commons licence and your intended use is not permitted by statutory regulation or exceeds the permitted use, you will need to obtain permission directly from the copyright holder. To view a copy of this licence, visit http://creativecommons.org/licenses/by/4.0/.

\section{Bibliografia}

1. Kwok R, Choi KC, Wong GL et al (2016) Screening diabetic patients for non-alcoholic fatty liver disease with controlled attenuation parameter and liver stiffness measurements: a prospective cohort study. Gut 65(8):1359-1368

2. Lombardi R, Airaghi L, Targher G et al (2020) Liver fibrosis by FibroScan independently of established cardiovascular risk parameters associates with macrovascular and microvascular complications in patients with type 2 diabetes. Liver Int 40(2):347-354 